\title{
Avaliação energética da maravalha gerada em uma serraria de pequeno porte
}

\author{
Jordão Cabral Moulin ${ }^{1 *}$, Marina Donária Chaves Arantes ${ }^{1}$, Fabrício Gomes Gonçalves ${ }^{1}$, \\ Juarez Benigno Paes ${ }^{1}$, Edy Eime Pereira Baraúna ${ }^{2}$ e Rafael Amorim Rosa ${ }^{1}$
}

${ }^{I}$ Departamento de Engenharia Florestal; Centro de Ciência Agrárias da Universidade Federal do Espírito Santo;29550-000; Jerônimo Monteiro - ES - Brasil. ${ }^{2}$ Curso de Engenharia Florestal; Universidade Federal do Tocantins; 77402-970; Gurupi - TO - Brasil.

\begin{abstract}
The work was aimed at making the quantification and qualification of the energy generated in a sawmill shavings in the city of Jeronimo Monterio/ES, this material was composed of a mixture of different woods. Technical visits were made at the mill twice a month with the intention of performing the quantitative analysis, which was calculated from a container known, and to collect three samples for the performance of moisture, bulk density, chemical analysis, ash content, elemental chemical analysis and calorific value. It was concluded that the bulk density of wood shavings is considered suitable for production of briquette, and from other analysis showed that this residue is suitable for energy production. The owner of the sawmill might use this material as an energy source, thus minimizing their spending, and prevent environmental damage.
\end{abstract}

Key words: Residue, quantification and qualification, energy

\section{INTRODUÇÃO}

O Brasil é um grande produtor de madeira e ao mesmo tempo um grande consumidor; conseqüentemente ocorre uma produção de 60 milhões de toneladas de resíduos de madeira por ano, e o País está entre os melhores exemplos de potencialidade para o uso de resíduos madeiráveis para a geração de energia (Brand, 2009).

De acordo com Teixeira e César (2006) o processo realizado da extração da árvore até a transformação da madeira no produto final desejado é gerido de forma ineficiente. Somado a isso, o descarte dos produtos madeiráveis no fim de sua vida útil demonstram a grande exploração dos recursos madeireiros, conseqüentemente levando a uma ampla geração de resíduos. No seguimento madeireiro, o aproveitamento de resíduos gerados pela extração e industrialização da madeira pode beneficiar desde indústrias de processamento primário até fábricas de móveis (Remade, 2008).

Conforme Brand et al. (2002) com a formação dos resíduos de madeira, pode surgir problemas como o assoreamento e poluição dos rios, poluição do ar por causa da queima dos mesmos, perda de espaço para o armazenamento, deixando também a serraria com aspecto sujo e propiciando maior índice de acidentes.

Para reduzir os impactos negativos que os resíduos causam, seria importante o Brasil criar uma legislação em que obrigue o gerador do resíduo dar um destino apropriado para os mesmos, ou que ocorra uma cobrança de taxas por quantidade de resíduos gerados, como é realizado em alguns países da Europa (Quirino, 2010). Além de ajudar a preservar o ambiente, poderia contribuir também para o reaproveitamento de resíduos, conseqüentemente podendo criar novos empregos. Existe uma grande importância nos estudos embasados em problemas inerentes à geração de resíduos como suas características e quantidades geradas, pois, maiores serão as chances de um uso mais adequado desses resíduos.

$\mathrm{O}$ presente estudo teve como objetivo realizar a quantificação e qualificação da maravalha gerada em uma serraria de pequeno porte para fins energéticos. Foram verificadas as seguintes características: umidade, densidade a granel, química e elementar, teor de cinzas, poder calorífico superior e o volume de maravalha gerado por mês na serraria.

Author for correspondence: jordao_cm@hotmail.com 


\section{MATERIAL E MÉTODOS}

A coleta do material foi executada na serraria $\mathrm{M}$. A. R. Perciliano - ME, localizada no município de Jerônimo Monteiro/ES, a pesquisa ocorreu durante o período de maio a outubro de 2010.

A empresa usa em seu processo produtivo, tanto madeiras de reflorestamento quanto madeiras nativas, sendo as principais: Eucalyptus sp.(eucalipto), Acacia polyphylla (acácia), Anadenanthera sp. (angico), Laurus nobilis (louro), Gallesia intergrifólia (pau d'alho) e Plathymenia sp. (vinhático). Os resíduos gerados dessas madeiras são armazenados juntos no pátio da serraria. Como os resíduos não são separados de acordo com a espécie que a originou, os materiais obtidos para a realização das análises foi uma mescla da maravalha dessas madeiras.

A escolha da maravalha para realização do experimento foi por causa da sua maior quantidade quando comparado aos outros resíduos gerados na serraria, este material é originado principalmente pelas operações na plaina e a desengrossadeira.

Ocorreram visitas técnicas à serraria duas vezes por mês, durante o período de cinco meses, com a finalidade de realizar a análise quantitativa e para a coleta de três amostras, as quais foram obtidas de diferentes locais da empresa. As datas em que foram realizadas as coletas foram nomeadas em ordem alfabética.

No Laboratório de Energia da Biomassa do Departamento de Engenharia Florestal do Centro de Ciências Agrárias da Universidade Federal do Espírito Santo - DEF/CCA/ES, realizou-se a análise de umidade segundo o método Brasileiro MB 26/40 da associação Brasileira de Normas Técnica - ABNT (1940) e teor de cinzas conforme M 11/77 a associação Brasileira Técnica de Celulose e Papel - ABCP (1977); e também a densidade a granel de acordo com a NBR 6922 (ABNT, 1983).

A análise química da maravalha procedeu-se segundo ABCP M/68 e Norma Tappi T204-05-76 (1998), no Laboratório de Química da Madeira do DEF/CCA/UFES.
O volume de resíduo gerado na serraria foi calculado, utilizando um recipiente de volume conhecido. Para o cálculo do volume mensal utilizou-se uma média das diferentes datas de coleta, em seguida multiplicado pelo número de dias que a empresa trabalha (22 dias/mês), esse cálculo pôde ser realizado em função do proprietário da empresa afirmar que a quantidade da maravalha gerada não varia ao longo do mês.

A composição elementar da maravalha foi determinada no Laboratório de Energia da Biomassa do Departamento de Engenharia Florestal da Universidade Federal de Lavras MG, como também o poder calorífico, em que foi obtido de acordo com a NBR 8633 (ABNT, 1983).

\section{RESULTADOS E DISCUSSÃO}

Análises dos resíduos de madeira

$\mathrm{Na}$ Tabela 1 são apresentados os valores médios de umidade (U), volume referente à data de coleta da maravalha $(\mathrm{V})$, densidade a granel (D), teor de cinzas (TCz) e o poder calorífico superior (PCS) encontrados para as respectivas amostras, submetidas ao teste de Tukey a nível de 5\% de significância. A única amostra que diferiu entre si para os valores de umidade, em nível de $5 \%$ de significância foi a amostra identificada com a letra $\mathrm{H}$, que apresentou o maior valor $(19,54 \%)$, isso ocorreu por causa da amostra ter sido coletada logo em seguida em que a madeira foi usinada, ou seja, esse material ficou exposto pouco tempo ao ambiente, ocasionando uma menor perda de umidade. No entanto as outras amostras apresentaram comportamento similar, fato ocorrido, por causa da maravalha ter sido coletada em um local coberto e com boa circulação de ar.

A umidade da madeira é uma característica importante que influência negativamente na produção de calor por unidade de massa do material. Para que a madeira esteja em boas condições para produção de energia, deve estar com umidade abaixo de 25\% (Vale et al., 2000). Desta forma, as amostras do presente estudo apresentaram valores de umidades propícias para produção de energia. 
Tabela 1. Valores médios de umidade, volume referente à data de coleta da maravalha, densidade a granel teor de cinzas e poder calorífico para as amostras de maravalha.

\begin{tabular}{|c|c|c|c|c|c|}
\hline Identificação & $\mathbf{U}(\%)$ & $\mathbf{V}\left(\mathbf{m}^{3}\right)$ & D $\left(\mathrm{Kg}^{-\mathrm{m}^{-3}}\right)$ & $\mathrm{TCz}(\%)$ & PCS $\left(\text { cal.g }^{-1}\right)^{*}$ \\
\hline $\mathbf{A}$ & $\begin{array}{l}10,57 \mathrm{~b} \\
(0,30)^{* *}\end{array}$ & 0,64 & $\begin{array}{c}73,86 \mathrm{bc} \\
(8,00)\end{array}$ & $\begin{array}{l}0,47 \mathrm{c} \\
(0,15)\end{array}$ & $\begin{array}{c}4.716,00 \\
(77,67)\end{array}$ \\
\hline B & $\begin{array}{c}10,77 \mathrm{~b} \\
(0,16)\end{array}$ & 0,70 & $\begin{array}{l}73,31 \mathrm{c} \\
(11,72) \\
\end{array}$ & $\begin{array}{l}1,07 \mathrm{~b} \\
(0,17)\end{array}$ & $\begin{array}{c}4.714,67 \\
(18,01)\end{array}$ \\
\hline $\mathbf{C}$ & $\begin{array}{c}10,61 \mathrm{~b} \\
(0,22)\end{array}$ & 0,90 & $\begin{array}{c}63,91 \mathrm{~cd} \\
(5,24)\end{array}$ & $\begin{array}{c}0,81 \mathrm{bc} \\
(0,03) \\
\end{array}$ & $\begin{array}{c}4.679,67 \\
(4,51) \\
\end{array}$ \\
\hline D & $\begin{array}{c}11,61 \mathrm{~b} \\
(0,13)\end{array}$ & 0,19 & $\begin{array}{c}52,52 \mathrm{~d} \\
(4,56) \\
\end{array}$ & $\begin{array}{l}1,00 \mathrm{~b} \\
(0,06)\end{array}$ & $\begin{array}{l}4.797,00 \\
(248,15) \\
\end{array}$ \\
\hline $\mathbf{E}$ & $\begin{array}{c}11,95 \mathrm{~b} \\
(0,18)\end{array}$ & 0,32 & $\begin{array}{c}94,90 \mathrm{a} \\
(2,89) \\
\end{array}$ & $\begin{array}{l}2,18 \mathrm{a} \\
(0,24) \\
\end{array}$ & $\begin{array}{c}4.659,67 \\
(14,47) \\
\end{array}$ \\
\hline $\mathbf{F}$ & $\begin{array}{c}11,42 \mathrm{~b} \\
(0,11)\end{array}$ & 0,83 & $\begin{array}{c}79,00 \mathrm{abc} \\
(3,51)\end{array}$ & $\begin{array}{l}1,03 \mathrm{~b} \\
(0,04)\end{array}$ & $\begin{array}{l}4.649,67 \\
(157,89) \\
\end{array}$ \\
\hline $\mathbf{G}$ & $\begin{array}{c}12,00 \mathrm{~b} \\
(0,44)\end{array}$ & 1,15 & $\begin{array}{c}90,84 \mathrm{ab} \\
(1,63)\end{array}$ & $\begin{array}{c}0,71 \mathrm{bc} \\
(0,07)\end{array}$ & $\begin{array}{c}4.714,33 \\
(13,87)\end{array}$ \\
\hline $\mathbf{H}$ & $\begin{array}{c}19,54 \mathrm{a} \\
(2,77)\end{array}$ & 0,77 & $\begin{array}{c}65,79 \mathrm{~cd} \\
(4,70)\end{array}$ & $\begin{array}{l}0,51 \mathrm{c} \\
(0,08)\end{array}$ & $\begin{array}{l}4.635,67 \\
(176,90)\end{array}$ \\
\hline
\end{tabular}

As médias seguidas por uma mesma letra, em cada coluna, não diferem estatisticamente (Tukey, $\mathrm{p}>0,05)$.

* Não significativo pelo teste de $\mathrm{F}(\mathrm{p}>0,05)$.

** Desvio padrão.

Como pode ser observado na Tabela 1 , o volume da maravalha variou de 0,19 a $1,15 \mathrm{~m}^{3}$, no entanto os baixos valores encontrados para as amostras identificadas com as letras $\mathrm{E}$ e F, podem ser explicado pelo fato da indisponibilidade do material na serraria, o que ocasionou no baixo volume. A quantidade mensal de maravalha foi em média $18,30 \mathrm{~m}^{3}$.

Dentre os valores encontrados, a amostra identificada como a letra D foi a que apresentou o menor valor de densidade a granel, sendo o valor de $52,52 \mathrm{~kg} \cdot \mathrm{m}^{-3}$, já a amostra identificada como a letra $\mathrm{E}$, foi a que apresentou o maior valor de densidade a granel, cujo valor foi de $94,90 \mathrm{Kg} . \mathrm{m}^{-3}$. Os valores de densidade encontrado no presente estudo, foram inferiores aos obtidas por Hillig et al. (2009), pois, os valores médios de densidade a granel encontrados em seus estudos foram: serragem com $223 \mathrm{~kg} . \mathrm{m}^{-3}$; serragem de madeira serrada com $216 \mathrm{~kg} \cdot \mathrm{m}^{-3}$. Possivelmente estes valores superiores de densidade a granel podem ser explicados pela utilização de serragem a qual possui uma menor granulometria, ocupando um maior espaço na caixa utilizada para determinação dos valores de densidade a granel, acarretando maior massa, e consequentemente uma maior densidade. Ribeiro e Machado (2005), também encontraram valores superiores para maravalha, sendo de $130 \mathrm{~kg} . \mathrm{m}^{-3}$.

Os baixos resultados encontrados referentes à densidade a granel no presente estudo podem ser explicados pelo fato da heterogeneidade do material e características dos resíduos, uma vez que quanto maior a granulometria do material, maior será os espaços vazios dentro do recipiente, ocasionado assim, uma menor massa, e conseqüentemente uma menor densidade.

Os valores encontrados de densidade para as amostras do presente estudo apresentaram valores relativamente baixos. Quirino (2010) afirma que madeira de densidade baixa é ideal para ser compactado, em função disto, a maravalha é adequada para produção de briquetes.

A utilização de uma mescla de diferentes espécies com proporções indeterminadas para realização da análise, explica o fato das amostras terem variado significativamente. Porém, todos os resultados encontrados para o teor de cinzas foram baixos, em média $0,97 \%$. As amostras diferiram entre si 
em nível de 5\% de significância para os valores de teor de cinzas.

Ribeiro e Machado (2005) obtiveram valores superiores de teor de cinzas dos resíduos de lascas, cascas e maravalha da madeira de Eucaliptus spp. Em que foram submetidas à carbonização de $400 \mathrm{e}$ $600^{\circ} \mathrm{C}$, os valores encontrados foram de 1,2 e $1,6 \%$ para as lascas, 2,0 e 2,1\% para as cascas, e para maravalha foi de 1,6 e 1,9\%. Pinheiro et al. (2004) também encontraram valores diferentes, em que os resultados do teor de cinzas dos resíduos variaram de 0 a 5\%. Os valores citados acima são relativamente altos, porém ainda estão dentro da faixa de teor de cinzas citado por Pereira et al. (2000), que comenta que a madeira é composta de 0,5 a $5 \%$ de cinzas. Entretanto, procura-se obter a menor quantidade possível de mineral, pelo fato de acarretar uma série de problemas. Nota-se então, que os valores obtidos na serraria de Jerônimo Monteiro, são aptos para produção de energia, pelo fato de apresentar um menor teor de cinzas.

Os valores de poder calorífico superior foram iguais estatisticamente para todas as amostras pelo teste F. Verifica-se que os valores encontrados por Pinheiro et al. (2004) foram entre 4000 a 5000 cal.g ${ }^{-1}$, como os valores do poder calorífico do presente estudo foram similares e altos, torna a maravalha mais adequada para produção de energia.

\section{Composição química dos resíduos}

Na Tabela 2 são apresentados os valores médios das análises químicas realizado nas amostras de maravalha, as quais foram submetidas ao teste de Tukey em nível de 5\% de significância. Como pode ser observado na amostra identificada com a letra $\mathrm{H}$ foi a que apresentou maior valor de lignina $(35,31 \%)$, o menor valor $(32,25 \%)$ foi verificado na amostra A. Couto (2009) obteve valores inferiores de lignina para a serragem de Eucalyptus sp., sendo o valor de $31,1 \%$. Paula et al. (2010) também obtiveram valores inferiores de lignina para os resíduos de serragem e de maravalha, sendo $20,88 \%$ e $20,62 \%$ respectivamente. Desta forma, os valores encontrados no presente estudo, são aptos para geração de energia. Pois, conforme Pimenta e Barcellos (2000) a madeira com alto teor de lignina, gera um carvão com maior poder calorífico e apresenta maior rendimento gravimétrico.

Para os valores de holocelulose, a amostra identificada com a letra B foi a que apresentou o menor valor, sendo $57,19 \%$, já a amostra identificada com a letra $\mathrm{E}$ apresentou o maior valor (62,26\%). Paula et al. (2010) encontrou valores superiores de holocelulose, tanto para serragem $(68,57 \%)$ como para maravalha $(73,65 \%)$. Couto (2009) também obteve valores superiores de holocelulose para a serragem de Eucalyptus sp. (65,4\%).

Como poder ser observado na Tabela 2 , às amostras diferiram entre si em nível de $5 \%$ de significância para os valores de extrativos, ocorrendo valores variados, de $3,20 \%$ a $9,24 \%$, notando assim, que havia na serraria materiais heterogêneos. Os valores da amostra identificada com a letra $\mathrm{A}$, foram próximos dos encontrados por Paula et al. (2010), que obtiveram para serragem de madeira o valor de $9,37 \%$ de extrativo, os mesmos autores encontraram 5,60\% de extrativo para maravalha, resultado próximo aos valores das amostras identificadas com a letra $\mathrm{F}, \mathrm{G}$ e H.

Segundo Klock et al. (2005), a madeira seca pode conter em torno de 3 a $10 \%$ de extrativos, os quais apresentam em sua composição os fenóis, que contribuem positivamente para o poder calorífico. Os valores encontrados no presente estudo estão dentro da faixa de teor de extrativo citado por este autor, sendo os valores relativamente altos, visto isso, as amostras estão adequadas para produção de energia, já que os extrativos contribuíram positivamente para a produção de energia por unidade de massa. 
Tabela 2. Valores médios de lignina, extrativos e holocelulose para as amostras.

\begin{tabular}{cccc}
\hline Identificação & Lignina (\%) & Holocelulose (\%) & Extrativo (\%) \\
\hline \multirow{2}{*}{$\mathbf{A}$} & $32,25 \mathrm{~b}$ & $58,51 \mathrm{ab}$ & $9,24 \mathrm{a}$ \\
& $(0,38)^{* *}$ & $(2,46)$ & $(2,19)$ \\
\hline \multirow{2}{*}{$\mathbf{B}$} & $34,24 \mathrm{ab}$ & $57,19 \mathrm{~b}$ & $8,57 \mathrm{ab}$ \\
& $(0,82)$ & $(1,57)$ & $(0,76)$ \\
\hline \multirow{2}{*}{$\mathbf{C}$} & $33,39 \mathrm{ab}$ & $58,68 \mathrm{ab}$ & $7,93 \mathrm{ab}$ \\
& $(0,44)$ & $(1,17)$ & $(1,34)$ \\
\hline \multirow{2}{*}{$\mathbf{D}$} & $32,36 \mathrm{~b}$ & $60,61 \mathrm{ab}$ & $6,77 \mathrm{ab}$ \\
& $(0,73)$ & $(1,27)$ & $(1,03)$ \\
\hline \multirow{2}{*}{$\mathbf{E}$} & $34,54 \mathrm{ab}$ & $62,26 \mathrm{a}$ & $3,20 \mathrm{c}$ \\
& $(1,47)$ & $(1,97)$ & $(0,54)$ \\
\hline \multirow{2}{*}{$\mathbf{F}$} & $33,57 \mathrm{ab}$ & $60,75 \mathrm{ab}$ & $5,67 \mathrm{bc}$ \\
& $(1,01)$ & $(1,78)$ & $(0,79)$ \\
\hline \multirow{2}{*}{$\mathbf{G}$} & $33,77 \mathrm{ab}$ & $60,18 \mathrm{ab}$ & $6,05 \mathrm{abc}$ \\
& $(0,26)$ & $(0,66)$ & $(0,44)$ \\
\hline \multirow{2}{*}{$\mathbf{H}$} & $35,31 \mathrm{a}$ & $58,73 \mathrm{ab}$ & $5,96 \mathrm{abc}$ \\
& $(1,04)$ & $(0,49)$ & $(1,50)$ \\
\hline
\end{tabular}

As médias seguidas por uma mesma letra, em cada coluna, não diferem estatisticamente (Tukey, >0,05).

** Desvio padrão.

\section{Análise elementar da maravalha}

Os valores da análise elementar para as respectivas amostras estão presentes na Tabela 3. Como pode ser observado os valores dos teores de nitrogênio variaram significativamente em nível de $5 \%$ de significância pelo teste de Tukey, o menor valor de $0,08 \%$ e o maior de $0,35 \%$ para as amostras $\mathrm{G}$ e $\mathrm{E}$. Segundo Klock et al. (2005), o valor máximo de nitrogênio presente na madeira é de $0,1 \%$, observando assim, $83,3 \%$ dos resultados estão acima desse valor. Couto (2009) obteve um alto valor de nitrogênio de $0,13 \%$. Vale ressaltar que o nitrogênio é prejudicial ao ser humano, por causa da liberação de óxidos de nitrogênio.

Os valores dos teores de carbono foram iguais estatisticamente para todas as amostras pelo teste F. Couto (2009) obteve 45,5\% de carbono para a serragem de Eucalyptus sp. Portanto, nota-se que todas as amostras coletadas possuem valores superiores. Em função disto, os valores do presente estudo estão propícios para geração de energia, já que o carbono contribui positivamente para o poder calorífico.

Os valores de hidrogênio não diferiram entre si em nível de 5\% de significância pelo teste F. Os valores variaram de $5,93 \%$ a $6,13 \%$, sendo próximos dos obtidos por Couto (2009), que encontrou o valor de $6,2 \%$ de hidrogênio para a da serragem de Eucalyptus sp..

Para os valores de enxofre, a amostra identificada pela letra $\mathrm{E}$ foi à única que diferiu em nível de $5 \%$ de significância no teste de Tukey, no entanto, os valores foram baixos, inferiores a $0,05 \%$. Couto (2009), obteve valores superiores $(0,07 \%)$ para resíduos de Eucalyptus sp..

As amostras identificadas pelas letras $\mathrm{F}$ e $\mathrm{H}$, foram às únicas que diferiram em nível de $5 \%$ de significância para os valores de oxigênio, as quais apresentaram os menores valores de oxigênio. Todas as amostras apresentaram valores inferiores de oxigênio, quando comparado com o resultado obtido para a serragem do Eucalyptus sp encontrado por Couto (2009), sendo esse valor de $48,1 \%$.

Os valores da relação carbono/hidrogênio como pode ser observado na Tabela 3 , foram iguais estatisticamente para todas as amostras pelo teste F. Esses resultados foram superiores aos obtidos por Couto (2009), para a serragem do Eucalyptus sp. $(7,34)$. Vale ressaltar que quanto maior a relação de carbono e hidrogênio, mais energético será o material. 
Tabela 3. Valores médios de nitrogênio, carbono, hidrogênio, enxofre, oxigênio, relação carbono/hidrogênio e carbono/nitrogênio para as amostras de maravalha.

\begin{tabular}{cccccccc}
\hline Identificação & $\mathbf{N}(\boldsymbol{\%})$ & $\mathbf{C ~ ( \% ) *}$ & $\mathbf{H}(\boldsymbol{\%}) *$ & $\mathbf{S}(\boldsymbol{\%})$ & $\mathbf{O}(\boldsymbol{\%})$ & $\mathbf{C / H}$ & $\mathbf{C} / \mathbf{N}$ \\
\hline \multirow{2}{*}{$\mathbf{A}$} & $0,19 \mathrm{~b}$ & 48,34 & 6,10 & $0,01 \mathrm{~b}$ & $45,37 \mathrm{a}$ & 7,92 & $264,53 \mathrm{~cd}$ \\
& $(0,03) * *$ & $(0,40)$ & $(0,05)$ & $(0,01)$ & $(0,45)$ & $(0,05)$ & $(48,49)$ \\
\hline \multirow{2}{*}{$\mathbf{B}$} & $0,16 \mathrm{bc}$ & 46,12 & 5,93 & $0,01 \mathrm{~b}$ & $47,79 \mathrm{a}$ & 7,78 & $286,53 \mathrm{bcd}$ \\
& $(0,02)$ & $(2,66)$ & $(0,27)$ & $(0,01)$ & $(2,85)$ & $(0,31)$ & $(47,10)$ \\
\hline \multirow{2}{*}{$\mathbf{C}$} & $0,14 \mathrm{bcd}$ & 47,83 & 6,06 & $0,00 \mathrm{~b}$ & $45,98 \mathrm{a}$ & 7,90 & $354,50 \mathrm{bc}$ \\
& $(0,02)$ & $(0,96)$ & $(0,06)$ & $(0,00)$ & $(1,04)$ & $(0,07)$ & $(44,79)$ \\
\hline \multirow{2}{*}{$\mathbf{D}$} & $0,11 \mathrm{~cd}$ & 48,17 & 6,05 & $0,01 \mathrm{~b}$ & $45,66 \mathrm{a}$ & 7,96 & $453,14 \mathrm{ab}$ \\
& $(0,03)$ & $(0,41)$ & $(0,06)$ & $(0,00)$ & $(0,49)$ & $(0,03)$ & $(95,12)$ \\
\hline \multirow{2}{*}{$\mathbf{E}$} & $0,35 \mathrm{a}$ & 46,18 & 5,94 & $0,05 \mathrm{a}$ & $47,49 \mathrm{a}$ & 7,77 & $133,95 \mathrm{~d}$ \\
& $(0,03)$ & $(1,29)$ & $(0,17)$ & $(0,01)$ & $(1,43)$ & $(0,01)$ & $(13,59)$ \\
\hline \multirow{2}{*}{$\mathbf{F}$} & $0,12 \mathrm{~cd}$ & 48,92 & 6,13 & $0,01 \mathrm{~b}$ & $44,82 \mathrm{ab}$ & 7,98 & $424,67 \mathrm{bc}$ \\
& $(0,02)$ & $(1,22)$ & $(0,18)$ & $(0,00)$ & $(1,39)$ & $(0,03)$ & $(61,57)$ \\
\hline \multirow{2}{*}{$\mathbf{G}$} & $0,08 \mathrm{~d}$ & 47,90 & 5,96 & $0,00 \mathrm{~b}$ & $46,07 \mathrm{a}$ & 8,04 & $627,02 \mathrm{a}$ \\
& $(0,01)$ & $(0,62)$ & $(0,60)$ & $(0,00)$ & $(0,68)$ & $(0,05)$ & $(45,11)$ \\
\hline \multirow{2}{*}{$\mathbf{H}$} & $0,12 \mathrm{bcd}$ & 47,54 & 5,97 & $0,00 \mathrm{~b}$ & $41,45 \mathrm{~b}$ & 7,97 & $402,08 \mathrm{bc}$ \\
& $(0,03)$ & $(0,98)$ & $(0,12)$ & $(0,00)$ & $(0,85)$ & $(0,08)$ & $(101,27)$ \\
\hline
\end{tabular}

N: nitrogênio; C: carbono; H: hidrogênio; S: enxofre; O: oxigênio; C/H: relação carbono e hidrogênio; C/N: relação carbono e nitrogênio.

As médias seguidas por uma mesma letra, em cada coluna, não diferem estatisticamente (Tukey, $p>0,05$ ).

* Não significativo pelo teste de F (p>0,05).

** Desvio padrão.

A relação de carbono/nitrogênio variou significativamente entre as amostras em nível de $5 \%$ de significância, em que o menor valor foi de 133,95 e o maior de 627,02. Alguns valores foram próximos dos obtidos por Santana (2009), tendo seus resultados variando de 374,77 a 543,67 . Os baixos valores encontrados para a relação indicam que o material é mais adequado para produção de energia, pois, segundo Schneider et al. (2005), quanto menor a relação carbono/nitrogênio, mais rápido o dióxido de carbono será emitido para o ambiente.

\section{CONCLUSÕES}

Com base no estudo realizado, conclui-se que a maravalha oriunda do processamento misto da madeira gerada em uma serraria de pequeno porte, é adequada à produção energética. Isso se deve, sobretudo:

Baixo teor de cinzas encontrados nas amostras;

Reduzida densidade a granel da maravalha;

Elevado teor de carbono presente no material, reduzido teor de enxofre e oxigênio;

Alto valor para os teores de lignina e extrativos;

Baixa umidade e alto poder calorífico do material;
Volume considerável de resíduo de maravalha gerado por mês pela serraria.

\section{RESUMO}

O trabalho teve como objetivo realizar a quantificação e qualificação energética da maravalha gerada em uma serraria do município de Jerônimo Monteiro/ES, esse material foi composto por uma mescla de diversas madeiras. Foram realizadas visitas técnicas na serraria duas vezes por mês com intuito da realização da análise quantitativa, e para a coleta de três amostras para a realização das análises de umidade, densidade a granel, análise química, teor de cinzas, análise elementar e o poder calorífico. Concluiu-se que a densidade a granel da maravalha analisada é apropriada para produção de briquete, verificou-se que este resíduo é adequado para a produção de energia.

Palavras-chave: Resíduo, quantificação e qualificação, energia

\section{REFERÊNCIAS}

ASSOCIAÇÃO BRASILEIRA DE NORMAS TÉCNICAS - ABNT. NBR MB 26. Rio de Janeiro. 1940. 
ASSOCIAÇÃO BRASILEIRA TÉCNICA DE CELULOSE E PAPEL - ABTCP. Normas técnicas. São Paulo. 1968.

ASSOCIAÇÃO BRASILEIRA TÉCNICA DE CELULOSE E PAPEL - ABTCP. Normas técnicas. São Paulo. 1977.

ASSOCIAÇÃO BRASILEIRA DE NORMAS TÉCNICAS - ABNT. NBR 8633. Rio de Janeiro. 1983.

ASSOCIAÇÃO BRASILEIRA DE NORMAS TÉCNICAS - ABNT. NBR 6922. Rio de Janeiro. 1983.

Brand, M. A.; Muñiz, G. I. B.; Da Silva, D. A.; Klock, U. (2002), Caracterização do rendimento e quantificação dos resíduos gerados em serraria através do balanço de materiais. Revista Floresta, 32, 247-259.

Brand, M. A. (2009), Resíduo: fonte de energia. Revista Referência, 87, 31-52.

Couto, G. M. Utilização da serragem de Eucalytus sp. na preparação de carvões ativados. Dissertação (Mestrado em Ciência e Tecnologia da Madeira) Universidade Federal de Lavras, 2009.

Hillig, E.; Schneider, V. E.; Pavoni, E. T. (2009), Geração de resíduos de madeira e derivados da indústria moveleira em função das variáveis de produção. Produção, 19,13-22.

Klock, U.; Muniz, G. I. B.; Hernandez, J. A. (2006), Química da madeira. Série didática, FUPEF, $86 \mathrm{p}$.

Paula, L. E. R.; Trugilho, P. F.; Rezende, R. N.; Baliza, A. E. R. (2010), Caracterização química de resíduos lignocelulósicos visando à produção de energia, In: XII Encontro Brasileiro em Madeiras e em Estruturas de Madeira. Lavras, 2010. Anais. Lavras: Instituto Brasileiro da Madeira e das Estruturas de Madeira. CD-ROM.

Pereira, J. C. D.; Sturion, J. A.; Higa, A. R.; Higa, R. C. V.; Shimizu, J. Y. (2000), Características da madeira de algumas espécies de Eucalipto plantadas no Brasil. Embrapa Informação tecnológica, $113 p$.

Pimenta, A. S. e Barcellos, D.C. (2000), Curso de atualização em carvão vegetal. Centro de Produções técnicas - CPT, 76p.

Pinheiro, G.; Rendeiro, G.; Pinho, J. (2004), Resíduos do setor madeireiro: aproveitamento energético. Biomassa \& Energia, 1, 199-208.

Quirino, W. F. (2010), Metodologia para analisar a viabilidade de usinas para compactação de resíduos. Instituto Brasileiro do Meio Ambiente e dos Recursos Naturais Renováveis - IBAMA, 35p.

Ribeiro, A. S. e Machado, A. A. de. (2005), Carbonização de resíduos do processamento mecânico da madeira de eucalipto. Ciência Florestal, 15, 1-7.

Santana, W. M. Efeito da idade e da classe diamétrica nas propriedades da madeira de Eucalyptus grandis e E. urophylla. Dissertação (Mestrado em Ciência e Tecnologia da Madeira) Universidade Federal de Lavras, 2009.

Schneider, P. R.; César Augusto Guimarães Finger, C. A. G.; Sobrinho, V. G.; Schneider, P. S. P. (2005), Determinação indireta do estoque de biomassa e carbono em povoamentos de Acácianegra (Acacia mearnsii De Wild.). Ciência Florestal, 15, 391-402.

TAPPI TECHNICAL DIVISIONS AND COMMITTEES. TAPPI Test Methods, Atlanta: Tappi Press, 1998.

Teixeira, M. G. e César, S. F. Produção de compósito com resíduo de madeira no contexto da ecologia industrial (2006), In: Encontro brasileiro em madeiras e em estruturas de madeira. São Pedro, 2006. Anais. São Pedro: Instituto Brasileiro da Madeira e das Estruturas de Madeira.

Vale, A. T. do.; Brasil, M. A. M.; Carvalho, C. M. de; Veiga, R. A. de A. (2000), Produção de energia do fuste de Eucalyptus grandis Hill exmaiden e Acacia mangium Willd em diferentes níveis de adubação. Revista Cerne, 6, 83-88. 